\title{
Neural Burst Firing and Its Roles in Mental and Neurological Disorders
}

\author{
Jie Shao ${ }^{1,2 t}$, Yunhui Liu ${ }^{1 \dagger}$, Dashuang Gao ${ }^{1,2}$, Jie Tu ${ }^{1,2}$, and Fan Yang ${ }^{1,2 *}$ \\ ${ }^{1}$ The Brain Cognition and Brain Disease Institute, Shenzhen Institute of Advanced Technology, Chinese Academy of \\ Sciences, Shenzhen-Hong Kong Institute of Brain Science-Shenzhen Fundamental Research Institutions, Shenzhen, China, \\ ${ }^{2}$ Shenzhen College of Advanced Technology, University of Chinese Academy of Sciences, Beijing, China
}

\section{OPEN ACCESS}

Edited by:

Barbara Jane Morley,

Boys Town National Research

Hospital, United States

Reviewed by:

Paul Geoffrey Overton,

The University of Sheffield,

United Kingdom

William Martin Connelly,

University of Tasmania, Australia

*Correspondence:

Fan Yang

fan.yang@siat.ac.cn

tThese authors have contributed equally to this work

Specialty section:

This article was submitted to

Cellular Neurophysiology,

a section of the journal

Frontiers in Cellular Neuroscience

Received: 14 July 2021

Accepted: 26 August 2021

Published: 27 September 2021

Citation:

Shao J, Liu Y, Gao D, Tu J and Yang $F$ (2021) Neural Burst Firing and Its Roles in Mental and Neurological

Disorders.

Front. Cell. Neurosci. 15:741292. doi: 10.3389/fncel.2021.741292
Neural firing patterns are critical for specific information coding and transmission, and abnormal firing is implicated in a series of neural pathologies. Recent studies have indicated that enhanced burst firing mediated by T-type voltage-gated calcium channels (T-VGCCs) in specific neuronal subtypes is involved in several mental or neurological disorders such as depression and epilepsy, while suppression of T-VGCCs relieve related symptoms. Burst firing consists of groups of relatively high-frequency spikes separated by quiescence. Neurons in a variety of brain areas, including the thalamus, hypothalamus, cortex, and hippocampus, display burst firing, but the ionic mechanisms that generating burst firing and the related physiological functions vary among regions. In this review, we summarize recent findings on the mechanisms underlying burst firing in various brain areas, as well as the roles of burst firing in several mental and neurological disorders. We also discuss the ion channels and receptors that may regulate burst firing directly or indirectly, with these molecules highlighted as potential intervention targets for the treatment of mental and neurological disorders.

Keywords: burst firing, ion channels, GPCRs, mental disorders, neurological disorders

\section{INTRODUCTION}

Neurons propagate action potentials with different inter-spike intervals, resulting in various firing patterns such as tonic and burst firing (Gerstner et al., 2014). In several brain regions, neurons can alter their firing pattern from tonic to bursting to regulate emotional states (Yang et al., 2018; Yuan et al., 2019), and abnormal burst firing is implicated in a series of neural pathologies (Sanabria et al., 2001; Fremont et al., 2014; Cain et al., 2018). Neuronal burst firing can enhance neural oscillations (Wang, 1999) and induce neural plasticity (Lisman, 1997; Sieber et al., 2013; Chan et al., 2016). Single burst stimulus given at different phase of $\theta$ oscillation can induce long-term potentiation or depression in CA1 in vitro (Huerta and Lisman, 1995). In midbrain dopamine (DA) neurons, burst firing stimulus promotes transmitter releasing, and the extracellular dopamine concentration increases in an exponential manner when the stimulation switched from tonic to burst (Gonon, 1988). Bursting promotes DA synaptic releasing but followed by rapid reuptake, which limits its diffusion into extrasynaptic space (Floresco et al., 2003). Moreover, compared with regular stimulation, burst firing evokes greater release of peptides like vasopressin and gonadotropinreleasing hormone (GnRH) in isolated brain tissue (Dutton and Dyball, 1979; Liu et al., 2011). Thus, burst firing appears to be critical in specific message coding and information transmission. Burst firing is not uncommon in the central nervous system (CNS), but its functions and underlying 
mechanisms vary with brain region and cell subtype. Burst firing in certain areas (especially the thalamus) is initiated by depolarization of low-threshold ion channels (e.g., T-VGCCs) and inward ion channels and is terminated by repolarization (Perez-Reyes, 2003). Thus, burst firing is highly dependent on membrane potential and ion channel dynamics, which offer potential targets for the manipulation of burst firing.

Many ion channels participate in generating or sustaining burst firing, including (but not limited to) voltage-dependent calcium (Cav), sodium (Nav), potassium (Kv) channels, calciumdependent potassium channels (SK/BK), and hyperpolarizationactivated cyclic nucleotide-gated cation ( $\mathrm{HCN}$ ) channels (Zhang et al., 2005; Cueni et al., 2008; Cain et al., 2015; Vandael et al., 2015). Several guanine nucleotide-binding proteins (G protein)coupled receptors (GPCRs) exert critical influence on neuronal bursting (Wolfe et al., 2003; Tirko et al., 2018). In this review, we discuss the ionic mechanisms and functions of burst firing in several brain areas. We also discuss the potential of intervention in neuronal burst firing via ion channel and receptor modulation as therapeutic treatment for certain neurological disorders.

\section{Ion Channels Underlying Burst Firing Burst Firing and T-VGCCs}

Among the ionic mechanisms underlying burst firing, the low-threshold response induced by T-VGCCs is a common cellular mechanism for initiating bursting in several brain regions(Cain and Snutch, 2010). The T-VGCC family consists of three widely expressed subtypes: Cav 3.1, Cav 3.2, and Cav 3.3 (Perez-Reyes, 2003; Cain and Snutch, 2011). Compared with other Cav channels, T-VGCC can be activated at more hyperpolarized membrane potentials (between -70 and $-60 \mathrm{mV}$ ) with slower inactivation kinetics (McRory et al., 2001; Perez-Reyes, 2003). These biophysical properties enable TVGCC-expressing neurons to be depolarized at low threshold (initial membrane potential should be hyperpolarized to ensure T-VGCC activation), and thus reach the threshold of Nav channels to fire a group of spikes over a short period before inactivation (Figure 1A). Repolarization channels, such as $\mathrm{Kv}$ and SK channels, are activated to rapidly hyperpolarize neurons back to their resting membrane potential, allowing T-VGCCs to recover from an inactive state and prepare for the next burst (Perez-Reyes, 2003). In this case, T-VGCC mediated bursting will produce pacemaker activity, which can affect neuronal oscillations. Despite their role in generating synchronous activity, T-VGCCs are also implicated in modulating synaptic connections. Low-threshold calcium spikes in the dendrites of thalamocortical relay neurons are essential for inhibitory postsynaptic long-term potentiation (Sieber et al., 2013). T-VGCCs in the dendrites of hippocampal CA1 neurons enable subthreshold synaptic potential to induce local depolarizing potential (Magee et al., 1995).

Many antagonists of T-VGCCs exert blockade of all three subtypes, and include nickel, mibefradil, and Z944 (Zamponi et al., 1996; Marks et al., 2016b; Yang et al., 2018). Considering subtype specificity, isoform blockade by nickel displays regional, tissue-dependent variability, and Cav3.2 show the highest sensitivity to nickel among isoforms (Zamponi et al., 1996;
Kang et al., 2006). Extracellular histidine residue of glutamine at position 191 of Cav 3.2 is a high-affinity nickel binding site, and ascorbate acts through this site to inhibit the inward calcium current by activating Cav 3.2 (Nelson et al., 2007). High antagonist sensitivity makes it easier to block Cav 3.2, but it is much more difficult to discriminate Cav3.1 and Cav3.3 from other isoforms. As such, greater effort should be expended on developing and designing drugs that specifically modulate each isoform. Lack of subtype-specific antagonists makes it difficult to determine the differences in the properties and functions of the isoforms. However, transient expression of the three subtypes in cultured cells indicates that the Cav3.3 isoform is activated and inactivated more slowly than the other two isoforms but at the most hyperpolarized thresholds. Furthermore, the Cav3.1 subtype exhibits the fastest activation and inactivation kinetics (Kozlov et al., 1999; McRory et al., 2001). In thalamic relay neurons displaying rebound-bursting, replacing T-VGCCs with Cav3.1 results in decreased firing rates, whereas substitution of Cav3.3 has no obvious effect on burst firing and Cav3.2 only produces one rebound spike (McRory et al., 2001). This suggests that the T-VGCC isoforms possess distinct electrophysiological properties and different contributions to the generation of bursts.

In addition to the distinct characteristics of the three isoforms, the properties of T-VGCC-mediated burst firing depend on the neuronal cell type in the different brain areas. Calcium currents conducted by T-VGCCs in GnRH neurons exhibit relatively slow inactivation kinetics, indicating that Cav3.3 may be the most important subtype for burst firing in these neurons (Zhang et al., 2009). In the thalamus, Cav3.1 is expressed in the ventrobasal and laterodorsal thalamic neurons, Cav3.3 is expressed in the laterodorsal and reticular parts, and Cav3.2 is expressed in the reticular thalamus only (Nelson et al., 2007). Cav3.2 knockout in dentate gyrus neurons impairs the ability to fire in a burst pattern (Dumenieu et al., 2018). Certain subpopulations in the medial habenula (MHB) exhibit burst firing, relying primarily on the function of Cav3.1 (Vickstrom et al., 2020). The fast kinetics of low threshold currents suggest the possible contribution of Cav3.1 to bursting in the subthalamic nucleus (Tai et al., 2011). Together, the variability in T-type currents in different brain regions may reflect differential expression patterns of Cav3 channel isoforms, which lead to regional specificity in $\mathrm{T}$ VGCC-induced burst firing.

T-VGCC expression is also highly related to burst firing. Previous research has shown that T-VGCC expression and burst firing in the ventral subiculum of stress-susceptible mice are enhanced after long periods of defeat-stress by aggressor mice, although the underlying mechanism remains unclear (Lee et al., 2019b). 17 $\beta$-Estradiol (E2) can modulate T-VGCC expression with subtype- and brain region-specificity through the estrogen receptor (Bosch et al., 2009). Following E2 administration, T-VGCCs are up-regulated in several hypothalamic nuclei, resulting in enhanced T-type calcium currents and rebound bursting. In the pituitary gland, Cav3.1 expression is increased by E2 application, and T-VGCC-mediated burst firing in the pituitary gland is considered to modulate hormone secretion (Qiu et al., 2006). E2 treatment also up-regulates the expression of the three T-VGCC subtypes in GnRH neurons, which, in turn, 
A
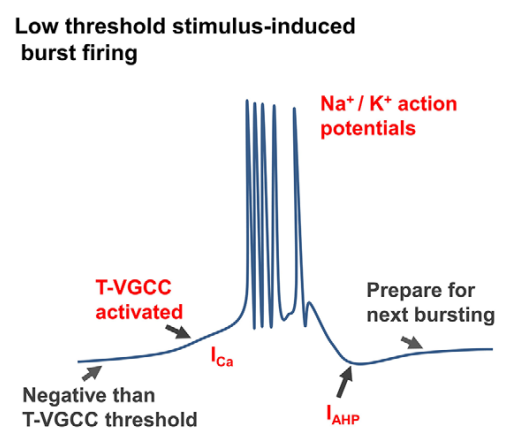

D

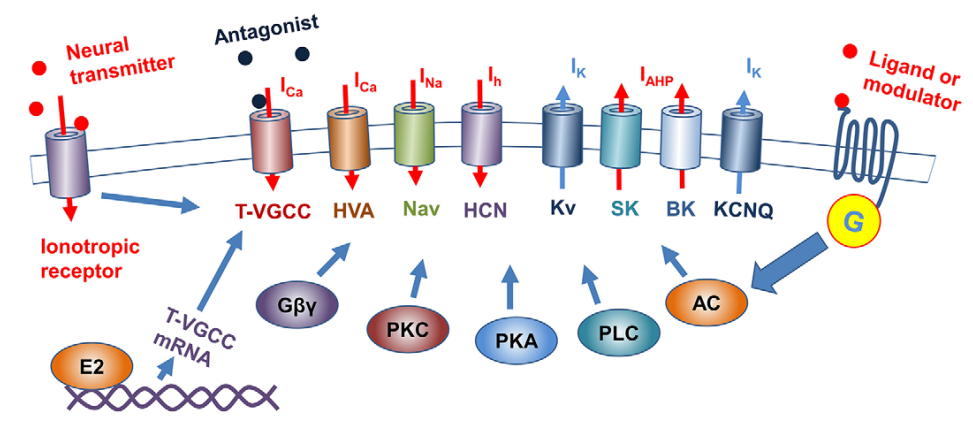

Rebound burst firing

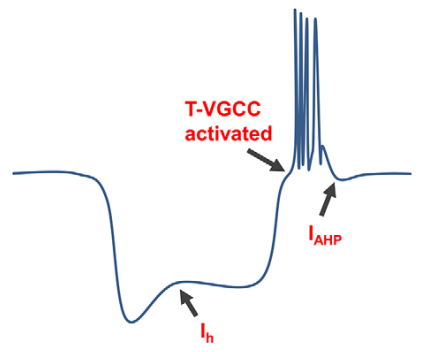

C

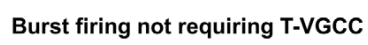

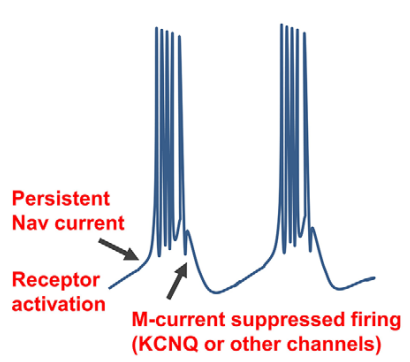

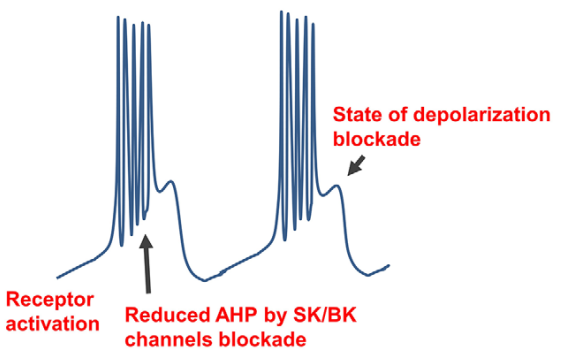

activation channels blockade

FIGURE 1 | Mechanisms underlying generation and regulation of neuronal burst firing. (A) Schematic of burst firing induced by T-VGCC activation caused by slight depolarization, T-VGCCs recover from inactivation by the after hyperpolarization (AHP) to prepare for next bursting. (B) Schematic of rebound burst firing, with T-VGCCs activated by hyperpolarization-activated cation current (Ih current) conducted by HCN channels. (C) Mechanisms underlying burst firing independent of T-VGCCs. Top: Slow inactivation, persistent Nav current induces repetitive action potential firing in hippocampus, M currents [mediated by channels such as Potassium Voltage-Gated Channel Subfamily Q Member (KCNQ)] inhibit membrane excitability, and participate in regulating of frequency and duration of burst firing. Bottom: Putative mechanism underlying burst firing of substantia nigra dopamine neurons induced by SK/BK channel inhibition in vitro. After excitation, when calcium-activated potassium channels are inhibited, membrane gradually reaches a state of depolarization blockade. (D) Mechanisms underlying burst firing regulation. Conductance of ion channels underlying burst firing is affected by membrane potential, antagonists, and G-protein-coupled (GPCR) and ionotropic receptor activity. Altered expression by E2 of T-VGCCs affects T-type currents, which directly regulates burst firing.

increases the T-type calcium current to promote burst firing (Zhang et al., 2009). Moreover, alternate splicing events during T-VGCC expression can result in changes in the T-type current, which may affect burst firing (Zhong et al., 2006). In summary, T-VGCC conductance and expression can be manipulated to regulate the function of burst firing.

\section{Burst Firing and Other lon Channels}

Other channels, including high threshold Cav channels, persistent Nav channels, SK/BK channels, and HCN channels, also modulate membrane potential to sustain burst firing or directly initiate burst firing (Carr et al., 2003; Zhang et al., 2005; Rouchet et al., 2008; Cain et al., 2015; Niday and Bean, 2021). However, these induced burst firings display different properties compared with T-VGCC-mediated bursting. Previous studies have reported that DA neurons in several areas display high-frequency bursts, although the underling mechanism is complex (Grace and Bunney, 1984; Zhang et al., 2005; Blythe et al., 2009): in the substantia nigra (SN), activation of Cav1 channels increases burst firing by inhibiting $\mathrm{K}^{+}$currents ; blocked of high voltage-gated Cav1.2 and Cav1.3 in the ventral tegmental area (VTA) significantly suppresses the bursting firing, while enhanced calcium influx conducted by these L-type (not T-type) channels induces burst firing directly. Many Kv channels also contribute to the regulation of burst firing, and their activation can hyperpolarize membranes and re-activate T-VGCCs (Perez-Reyes, 2003). The slow inactivation kinetics of persistent Nav channels enables the shaping of repetitive firing in the hippocampus (Carr et al., 2003). Activation of oxytocin receptors can induce burst firing in the hippocampal CA2 area, with the duration and spiking rate of bursting modulated by the dynamics of the KCNQ (Potassium Voltage-Gated Channel Subfamily Q Member) and persistent Nav channels (Tirko et al., 2018). Furthermore, activation of metabotropic glutamate receptor depolarizes SN DA neuronal membranes and induces firing, as well as reduced activity of small conductance SK channels, can shift this regular firing into bursting in vitro (Prisco et al., 2002). Similar effects of SK channel blockade on burst firing of CA2 neurons have been observed in some serotoninergic neurons in the dorsal raphe (Rouchet et al., 2008). Inhibiting large conductance (BK) calcium-activated potassium channel currents in acutely isolated Purkinje neurons can convert the tonic firing of single action potentials into burst firing (Figure 1C; Niday and Bean, 2021). However, burst 
firings induced by SK/BK channel inhibition have mainly been performed mainly in vitro with pharmacological tools, and whether these channels facilitate burst firing in vivo requires more evidence.

The HCN channel is an important pacemaker and modulator of cardiac and neuronal excitability, and contributes to bursting in many neurons, especially rebound burst firing (Figure 1B; Cain et al., 2015). In genetic absence epilepsy rats from the Strasbourg (GAERS) model, ventrobasal thalamic neurons display an inherent suppression of burst firing, concomitant with an enhanced basal Ih current (Cain et al., 2015). Hyperpolarization of membrane potential activates HCN channels to produce Ih currents and slightly depolarize the membrane. Due to the nature of HCN channels, the enhancement of Ih currents in GAERS ventrobasal thalamic neurons inhibits hyperpolarization, and thus they require a significantly larger hyperpolarized current injection to de-inactivate T-VGCCs and produce rebound burst firing (Cain et al., 2015). Consistent with this, a decrease in the HCN1 channel is reported to occur in cortical neuron dendrites in the GAERS model, which contributes to calcium electrogenesis and neuronal bursting(Kole et al., 2007). However, ZD7288 treatment (antagonist of HCN channel) exerts an obvious inhibitory effect on burst firing in lateral habenula (LHB) neurons(Yang et al., 2018). Although the mechanism underlying $\mathrm{HCN}$ regulation of bursting is complex, interactions with the T-type calcium window and SK channel currents are considered to dominantly affect burst firing.

\section{GPCRs Involved in Regulation of Burst Firing}

Burst firing is tightly related to the activation and inactivation kinetics of ion channels like T-VGCCs (Nelson et al., 2007). In the CNS, GPCRs [such as metabotropic glutamate receptors (mGluRs)] bind with neurotransmitters or neuromodulators to activate downstream protein kinases, and thereby phosphorylate various ion channels and alter neuronal electrophysiological properties (Reiner and Levitz, 2018). These properties hint at the potential mechanism underlying the regulation of burst firing by GPCRs. Indeed, many studies have reported that neurotransmitters and neuromodulators can alter neuronal firing properties (Cantrell et al., 1996; Tirko et al., 2018) and transform firing pattern from tonic to bursting. Here, we explore the involvement of GPCRs in regulating burst firing; and discuss the possible treatment of burst firing-induced malfunctions by GPCR function manipulation.

\section{GPCRs Regulate T-VGCC-Mediated Burst Firing}

GPCRs can directly modulate T-VGCCs to regulate burst firing in many cell subtypes. Activation of mGluRs in cerebellar Purkinje neurons promotes T-VGCC currents (mainly Cav3.1), which rely on tyrosine phosphatase/kinase to enhance Cav3.1 conductance (Hildebrand et al., 2009). However, blockade of the mGluR1-phospholipase C beta4 (PLC beta4) pathway in thalamocortical neurons promotes T-VGCC currents and thus enhances burst firing (Cheong et al., 2008). Activation of corticotropin-releasing factor receptor 1 inhibits
Cav3.2 through $G$ protein $\beta \gamma$ subunits (Tao et al., 2008), mainly via binding to intracellular loop linking transmembrane domains (Wolfe et al., 2003). Serotonin (5- $\mathrm{HT}_{7}$ ) receptors can affect adenylyl cyclase via the $\mathrm{G}_{s \alpha}$ protein. In rats, 5-HT application in the adrenal glands activate $5-\mathrm{HT}_{7}$ receptors and enhances adenylyl cyclase activity, which increases T-type calcium currents (Lenglet et al., 2002). However, activation of the $5-\mathrm{HT}_{2}$ receptors in olivary neurons is reported to inhibit calcium influx through T-VGCCs (Placantonakis et al., 2000), with the same inhibition observed in T-VGCC-induced burst firing in subiculum neurons after activation of $5-\mathrm{HT}_{2} \mathrm{C}$ receptors (Petersen et al., 2017). The 5- $\mathrm{HT}_{2 \mathrm{C}}$ receptors belong to GPCRs coupled to $G_{\alpha q}$ and $G_{\beta \gamma}$. Activation of $G_{\alpha q}$-coupled neurokinin 1 receptor and related protein kinase $\mathrm{C}(\mathrm{PKC})$ and phospholipase C (PLC) inhibits Cav3.2 channels in cultured cells(Rangel et al., 2010), and $G_{\beta \gamma}$ subunits also suppress Cav3.2 conductance, as mentioned above. Taken together, this evidence indicates that activation of GPCRs affects the dynamics of T-VGCCs with neuronal subtype specificity.

\section{GPCRs Regulate Persistent Nav Current-Mediated Burst Firing}

Persistent Nav currents induce repetitive burst firing in many brain regions (Figure 1C), especially the hippocampus (Azouz et al., 1996; Carr et al., 2003). The hippocampus receives abundant cholinergic signals from the upstream basal forebrain (Frotscher and Léránth, 1985), and activity of muscarinic receptors is associated with the firing mode of the hippocampus neuronal subpopulations (Benardo and Prince, 1982). Muscarinic receptors belong to GPCRs (Bonner et al., 1987), and activation of muscarinic receptors with different concentrations of agonists exerts complicated effects on neuronal firing patterns (Benardo and Prince, 1982; Alroy et al., 1999). Application of low-concentration agonists to muscarinic receptors releases second messengers and activates PKC(Caulfield, 1993), which inhibits persistent Nav currents and related burst firing. In this scenario, the low concentrations of muscarinic receptor agonists switch the firing patterns of hippocampal CA1 neurons from bursting to consecutive tonic firing (Azouz et al., 1994; Alroy et al., 1999).

In contrast, several hippocampal-based studies have shown enhancement of bursting activity by muscarinic receptor activation (Benardo and Prince, 1982; Kawasaki and Avoli, 1996). These enhanced effects may be due to the PKC-dependent suppression of repolarized $\mathrm{K}^{+}$currents induced by the application of muscarinic agonists at high concentration, thereby promoting inward $\mathrm{Ca}^{2+}$ currents and burst firing (Brown et al., 1997). The activation of voltage-dependent cationic non-selective currents by muscarinic agonists may be another potential mechanism (Haj-Dahmane and Andrade, 1996). However, the concentration of agonist used in the above studies may exceed the physiological concentration of acetylcholine released by cholinergic terminals, and the enhanced effects are mainly induced by affecting membrane potential. Thus, burst firing mediated by persistent Nav currents in hippocampus may be inhibited by cholinergic signals under physiological conditions. 
Oxytocin is reported to play a critical role in modulating social behavior (Jones et al., 2017; Anpilov et al., 2020), with most effects initiated through the oxytocin receptor (GPCR binding with $\mathrm{G}_{\alpha \mathrm{i} / \mathrm{q}}$ proteins; Jurek and Neumann, 2018). In the hippocampal CA2 area, activation of oxytocin receptors by specific agonist [Thr4, Gly7]-oxytocin (TGOT) can induce pyramidal neuronal firing in bursting pattern. Persistent Nav channels are involved in the generation of burst firing and regulated by TGOT-induced PKC activation. Furthermore, inhibition of KCNQ by TGOT provides sustained depolarization to drive burst firing (Tirko et al., 2018).

Collectively, the above studies demonstrate that GPCRs exert brain region- and cell subtype-specific effects on T-VGCCs and associated ion channels to modulate burst firing (Figure 1D). However, exploration of GPCRs as potential targets to intervene in abnormal burst firing is still lacking. Due to the complicated relationship between GPCRs and burst firing, future research should focus on the possibility of manipulating specific GPCRs to rescue burst firing-induced malfunctions.

\section{Function of Burst Firing in Specific Brain Regions and Its Role in Neurological Disease \\ Physiological Functions of Burst Firing in Specific Brain Regions}

Under normal physiological conditions, burst firing can be found in various brain areas, such as the hypothalamus, thalamus, hippocampus, and cortex; however, its functions vary in different areas and cell subtypes. In the hypothalamus, GnRH neurons display T-VGCC-dependent burst firing, which can promote a GnRH and luteinizing hormone surge (Zhang et al., 2009). The ventromedial nucleus of the hypothalamus (VMH) shows high expression of T-VGCCs (Qiu et al., 2006), and oscillation activity in the VMH is tightly correlated with oscillation of sympathetic nerve activity (Iigaya et al., 2017). Superfusion with anorexigenic peptides, such as insulin, increases the spiking rate of VMH oscillations, while application of orexigenic peptides like leptin decreases oscillation frequency (Iigaya et al., 2019). These metabolism-related peptides may affect oscillation via depolarizing/hyperpolarizing the neuronal membrane, but the effect of altered oscillation on the sympathetic system is unignorable. These findings indicate that burst firing may be important in the hypothalamic regulation of secretion and metabolism.

In the thalamus, neuronal firing patterns are highly related to distinct sensory messages propagated to the cortex (Cheong et al., 2008). The pacemaker activity of the thalamic reticular nucleus induced by T-VGCC-mediated burst firing contributes to the appearance of oscillating spindles during non-rapid eye movement (Halassa et al., 2011). In thalamocortical neurons, increased bursting and decreased tonic firing reduce visceral pain responses (Cheong et al., 2008), contrary to the function of burst firing in dorsal root ganglia sensory neurons, which amplify peripheral pain signals (Jagodic et al., 2007).

In the hippocampus, the subiculum receives outputs from the hippocampal CA1 and thus regulates memory formation and spatial information processing (Joksimovic et al., 2017). T-VGCCs, especially Cav3.1, are abundantly expressed in subiculum neurons, which contributes to the generation of neuronal burst firing. Neurons in the subiculum that bursting infrequently exhibit stronger spatial modulation than those neurons that predominantly show burst firing (Simonnet and Brecht, 2019). Activation of oxytocin receptors in CA2 neurons enhances the excitatory-inhibitory ratio and plasticity of synapses connected to CA1 neurons, which are crucial for social memory processing and spatial representation (Tirko et al., 2018).

As the release of DA is associated with spiking rate, burst firing of midbrain DA neurons (including the $\mathrm{SN}$ and ventral tegmental area) in response to reward-predictive stimuli or reward better than expected significantly increases terminal DA phasic release in downstream targets like the nucleus accumbens and prefrontal cortex, and therefore promotes the motivation of reward-seeking and reward-driven learning (Schultz, 1998, 2002; Floresco et al., 2003). In this case, burst firing of DA neurons may code this prediction error and reinforces learning by modifying DA synaptic transmission. Burst firing and burst synchronization in the prefrontal and anterior cingulate cortices promote attention-focusing (Womelsdorf et al., 2014). Together, these studies highlight the critical role of burst firing in the maintenance of normal function of various neuronal subtypes.

\section{Burst Firing and Related Diseases in CNS}

Increasing evidence indicates that certain neuronal subpopulations display abnormal burst firing or shift firing patterns from tonic to burst firing in several nuclei during pathophysiological progress of certain neurological diseases.

Anxiety is an emotion state defined as the anticipation of future or potential threat and stress. Normal adaptive anxiety can promote individual survival, however, excessive pathological anxiety will have detrimental effects on the mind and body and requires clinical treatment (Crocq, 2015). Abnormal burst firing is reportedly involved in the pathophysiological processes of anxiety. In the hypothalamic paraventricular nucleus, exposure to chronic stress promotes neuronal burst firing and anxiety-like behavior, while reward prevents these effects (Yuan et al., 2019). Short bursts of spiking in dorsal raphe serotoninergic neurons enhances the release of serotonin (Gartside et al., 2000), which is critical for the regulation of anxiety state (Hornung, 2003). T-type calcium currents regulate excitability of medial amygdala neurons by inducing burst firing, and bursting in these neurons is significantly enhanced in rats under an anxiety state induced by spinal nerve ligation (Jiang et al., 2014; Padilla et al., 2016). The VMH is suggested to regulate anxiety-like behavior (Kunwar et al., 2015; Cho et al., 2020). However, whether TVGCCs, which are located in the VMH (Qiu et al., 2006) mediate burst firing in the $\mathrm{VMH}$ and regulate anxiety requires further investigation.

Depression is a mood disorder characterized by loss of interest in activity, accompanied by increased risk of suicidal behavior and psychiatric and psychosocial morbidity (Birmaher et al., 2002). The LHB is suggested to be an important node in regulating depression and related aversive behavior ( $\mathrm{Li}$ et al., 
2013). In animal models of depression, LHB neurons display increased burst firing, which is predicted to promote transmitter release to downstream brain regions (Yang et al., 2018).

LHB burst firing relies on T-VGCC dynamics (Yang et al., 2018). Based on the nature of T-VGCCs, LHB burst firing could potentially be manipulated through hyperpolarizing or depolarizing membrane potentials. Optogenetic hyperpolarization of LHB neurons can induce rebound burst firing, while knockdown of astroglia Kir4.1 can slightly depolarize the LHB neuronal membrane to suppress T-VGCCs (Cui et al., 2018). In addition, increased T-VGCC expression in the ventral subiculum can occur after long-term social defeat (especially in the nucleus accumbens-projecting subpopulation), and enhanced T-VGCC-mediated bursting may contribute to the development of depression (Lee et al., 2019a,b). These studies suggest a close link between depression and T-VGCC-mediated burst firing, and blockade of T-VGCC or suppression of T-VGCC-mediated burst firing could be a potential therapy for depression.

Epilepsy refers to several neurological disorders characterized by recurrent seizure, with excessive excitation or hypersynchronous neural activity (Fisher et al., 2014). Many factors may contribute to excessive and synchronous neural firing during epileptic seizure, such as reduced inhibitory activity or abnormal function of certain ion channels (Rowley et al., 2012; Wei et al., 2017). T-VGCC activation produces a low-threshold calcium potential, which initiates a depolarization cascade of neurons and generates high-frequency burst firing (Joksimovic et al., 2017). This burst firing is predicted to generate the hyper-synchronization of neurons and spread oscillations which promote absence seizure (Cain et al., 2018). The three T-VGCC subtypes are widely expressed in thalamic and thalamocortical neurons with subtype-specificity (Khosravani et al., 2004; Cain et al., 2018). Previous studies have suggested that T-VGCCs blocked in the thalamus suppress the neuronal synchronous firing that underlies absence seizures (Tringham et al., 2012). Activation of the thalamic reticular nucleus in vivo promotes the rebound burst firing in thalamocortical relay neurons (Halassa et al., 2011), this T-VGCC-mediated burst firing is critical for the genesis of synchronous spike-and-wave discharges during absence seizure (Kim et al., 2001). Together, T-VGCC-mediated bursting in these neurons is tightly related to the genesis of absence seizure.

Based on the relationship between T-VGCCs and absence seizure, antagonists of T-VGCCs are considered as potential anti-epileptic drugs. Long-term oral application of ethosuximide suppresses seizures in many rat models of absence epilepsy, including Wistar albino Glaxo/Rijswijk (WAG/Rij) and GAERS model rats, and its anti-epileptic effects mainly act via inhibition of T-VGCCs (Blumenfeld et al., 2008; Dezsi et al., 2013). In addition, application of T-VGCC antagonist Z944 rescues impaired visual recognition memory in the GAERS model (Marks et al., 2016a). These studies suggest the potential use of selective antagonists of T-VGCCs in the clinical intervention of absence epilepsy and related syndromes. However, although certain evidence indicates that T-VGCCs in the hippocampus may contribute to the epileptogenesis of temporal lobe epilepsy
(TLE), which is caused by abnormal structural or metabolic conditions (Sanabria et al., 2001; Becker et al., 2008), whether T-VGCC antagonists can effectively ameliorate TLE require further study.

Parkinson's disease $(P D)$ is a progressive neurodegenerative disease manifested with core motor symptoms like tremor. Oscillation due to central neuronal pacemakers is considered an important mechanism underlying rhythmic oscillatory activity in such tremors (Deuschl et al., 2001). Specifically, burst firing in inferior olivary neurons can transmit through the cerebellum and thus exert an important influence on the control of movement, with this oscillatory behavior determined by T-VGCC expression (Handforth et al., 2010; Bazzigaluppi and de Jeu, 2016). Administration of the T-VGCC antagonist zonisamide in PD animal models suppresses tremulous jaw movements which are postulated to affect olivocerebellar system rhythmicity (Miwa et al., 2011). Furthermore, enhanced neuronal burst firing in the STN is implicated in the pathology of $\mathrm{PD}$, and application of T-VGCC antagonists rescues motor disabilities in PD rats induced by 6-hydroxydopamine injection (Tai et al., 2011). Moreover, neurons in deep cerebellar nuclei exhibit bursting activity during rapid-onset dystonia parkinsonism, while Nav channel inhibition alleviates this dystonia (Fremont et al., 2014). Blockade of Cav1 channels partially rescues SN DA neurons in PD by inhibiting burst firing (Blythe et al., 2009). The above studies demonstrate that burst firing manipulation may be a possible treatment for PD tremor.

Other diseases are also related to neuronal burst firing. Several mental disorders, such as schizophrenia and addiction are tightly associated with abnormal neural activity of the DA neurons, while cholinergic input to DA neurons exerts an important influence on their firing pattern (Schultz, 2002; Zhang et al., 2005); however, the direct connection between burst firing and mental disorders like schizophrenia needs further study. In animal models of addiction, burst firing of VTA DA neurons alters transmitter release into reward-regulating downstream regions such as nucleus accumbens (Schultz, 2002; Floresco et al., 2003). However, the link between addiction and burst firing remains elusive. Moreover, the social behavior impairment of schizophrenia (Penn et al., 2008) is thought to be related to CA2 neuronal reduction (Benes et al., 2008). As such, manipulation of burst firing through oxytocin receptors in CA2 may facilitate neural circuits to restore the malfunctions of social behavior manifested by schizophrenia and other diseases (Wang, 1999). Genetic mutation of Cav3.2 is reported to result in a reduction in T-type currents, which may be involved in autism spectrum disorder (Splawski et al., 2006). In conclusion, these studies suggest the important role of neuronal burst firing in the pathology of many neurological diseases. However, further research is required to test the feasibility of antagonist and GPCR application in modulating burst firing for the treatment of such diseases.

\section{CONCLUSIONS}

Burst firing is a common pattern in the CNS, however the ionic mechanism of burst firing varies in different neuronal 
subtypes. T-VGCC activation-induced low-threshold spiking is the most common and important mechanism initiating neuronal burst firing, but its properties vary depending on the differentially expressed isoforms of T-VGCCs. Other channels, e.g., persistent $\mathrm{Nav}$ and KCNQ channels, also contribute to burst firing generation and maintenance in several neuronal subtypes. Burst firing in different neuronal subtypes displays distinct functions, and abnormal burst firing caused by changes in T-VGCC conductance (or that of other channels) is thought to be involved in the pathology of several neurological and physical diseases, including anxiety, depression, and epilepsy. Importantly, antagonists and GPCRs affecting those ion channels can regulate neuronal burst firing, suggesting potential application for the clinical treatment of anxiety, depression, epilepsy, and other burst firing-induced syndromes. Moreover, neurons in many brain regions exhibit burst firing (Qiu et al., 2006) but lack detailed exploration. Thus, clarifying the specific

\section{REFERENCES}

Alroy, G., Su, H., and Yaari, Y. (1999). Protein kinase C mediates muscarinic block of intrinsic bursting in rat hippocampal neurons. J. Physiol. 518, 71-79. doi: 10.1111/j.1469-7793.1999.0071r.x

Anpilov, S., Shemesh, Y., Eren, N., Harony-Nicolas, H., Benjamin, A., Dine, J., et al. (2020). Wireless optogenetic stimulation of oxytocin neurons in a semi-natural setup dynamically elevates both pro-social and agonistic behaviors. Neuron 107, 644-655. doi: 10.1016/j.neuron.2020.05.028

Azouz, R., Jensen, M. S., and Yaari, Y. (1994). Muscarinic modulation of intrinsic burst firing in rat hippocampal neurons. Eur. J. Neurosci. 6, 961-966. doi: 10.1111/j.1460-9568.1994.tb00590.x

Azouz, R., Jensen, M. S., and Yaari, Y. (1996). Ionic basis of spike afterdepolarization and burst generation in adult rat hippocampal CA1 pyramidal cells. J. Physiol. 492, 211-223. doi: 10.1113/jphysiol.1996.sp021302

Bazzigaluppi, P., and de Jeu, M. T. (2016). Heterogeneous expression of T-type $\mathrm{Ca}(2+)$ channels defines different neuronal populations in the inferior olive of the mouse. Front. Cell. Neurosci. 10:192. doi: 10.3389/fncel.2016.00192

Becker, A. J., Pitsch, J., Sochivko, D., Opitz, T., Staniek, M., Chen, C.-C., et al. (2008). Transcriptional upregulation of Cav3.2 mediates epileptogenesis in the pilocarpine model of epilepsy. J. Neurosci. 28, 13341-13353. doi: 10.1523/JNEUROSCI.1421-08.2008

Benardo, L. S., and Prince, D. A. (1982). Cholinergic excitation of mammalian hippocampal pyramidal cells. Brain Res. 249, 315-331. doi: 10.1016/00068993(82)90066-x

Benes, F. M., Lim, B., Matzilevich, D., Subburaju, S., and Walsh, J. P. (2008). Circuitry-based gene expression profiles in GABA cells of the trisynaptic pathway in schizophrenics versus bipolars. Proc. Natl. Acad. Sci. U S A 105, 20935-20940. doi: 10.1073/pnas.0810153105

Birmaher, B., Arbelaez, C., and Brent, D. (2002). Course and outcome of child and adolescent major depressive disorder. Child Adolesc. Psychiatr. Clin. N. Am. 11, 619-637. doi: 10.1016/s1056-4993(02)00011-1

Blumenfeld, H., Klein, J. P., Schridde, U., Vestal, M., Rice, T., Khera, D. S., et al. (2008). Early treatment suppresses the development of spike-wave epilepsy in a rat model. Epilepsia 49, 400-409. doi: 10.1111/j.1528-1167.2007.01458.x

Blythe, S. N., Wokosin, D., Atherton, J. F., and Bevan, M. (2009). Cellular mechanisms underlying burst firing in substantia nigra dopamine neurons. J. Neurosci. 29, 15531-15541. doi: 10.1523/JNEUROSCI.2961-09.2009

Bonner, T. I., Buckley, N. J., Young, A. C., and Brann, M. R. (1987). Identification of a family of muscarinic acetylcholine receptor genes. Science 237, 527-532. doi: 10.1126/science.3037705

Bosch, M. A., Hou, J., Fang, Y., Kelly, M. J., and Rønnekleiv, O. K. (2009). 17Beta-estradiol regulation of the mRNA expression of T-type calcium channel subunits: role of estrogen receptor alpha and estrogen receptor beta. J. Comp. Neurol. 512, 347-358. doi: 10.1002/cne.21901 functions and underlying mechanisms of burst firing in the CNS could facilitate treatment of these diseases.

\section{AUTHOR CONTRIBUTIONS}

JS and YL wrote the draft of the manuscript. FY designed, wrote, checked, and finalized the manuscript. All authors contributed to the article and approved the submitted version.

\section{FUNDING}

This work was supported by the National Natural Science Foundation of China (31800881 and 82072489), Key Research Program of Frontier Sciences of Chinese Academy of Sciences (QYZDB-SSW-SMC056), and Shenzhen Governmental Basic Research Grant (JCYJ20180507182301299, JCYJ20200109115405930 and JCYJ20200109150717745).

Brown, D. A., Abogadie, F. C., Allen, T. G., Buckley, N. J., Caulfield, M. P., Delmas, P., et al. (1997). Muscarinic mechanisms in nerve cells. Life Sci. 60, 1137-1144. doi: 10.1016/s0024-3205(97)00058-1

Cain, S. M., and Snutch, T. P. (2010). Contributions of T-type calcium channel isoforms to neuronal firing. Channels (Austin) 4, 475-482. doi: 10.4161/chan.4. 6.14106

Cain, S. M., and Snutch, T. P. (2011). Voltage-gated calcium channels and disease. Biofactors 37, 197-205. doi: 10.1002/biof.158

Cain, S. M., Tyson, J. R., Choi, H.-B., Ko, R., Lin, P. J. C., LeDue, J. M., et al. (2018). CaV3.2 drives sustained burst-firing, which is critical for absence seizure propagation in reticular thalamic neurons. Epilepsia 59, 778-791. doi: 10.1111/epi.14018

Cain, S. M., Tyson, J. R., Jones, K. L., and Snutch, T. P. (2015). Thalamocortical neurons display suppressed burst-firing due to an enhanced Ih current in a genetic model of absence epilepsy. Pflugers Arch. 467, 1367-1382. doi: 10.1007/s00424-014-1549-4

Cantrell, A. R., Ma, J. Y., Scheuer, T., and Catterall, W. A. (1996). Muscarinic modulation of sodium current by activation of protein kinase C in rat hippocampal neurons. Neuron 16, 1019-1026. doi: 10.1016/s08966273(00)80125-7

Carr, D. B., Day, M., Cantrell, A. R., Held, J., Scheuer, T., Catterall, W. A., et al. (2003). Transmitter modulation of slow, activity-dependent alterations in sodium channel availability endows neurons with a novel form of cellular plasticity. Neuron 39, 793-806. doi: 10.1016/s0896-6273(03) 00531-2

Caulfield, M. P. (1993). Muscarinic receptors--characterization, coupling and function. Pharmacol. Ther. 58, 319-379. doi: 10.1016/0163-7258(93) 90027-b

Chan, H. K., Yang, D.-P., Zhou, C., and Nowotny, T. (2016). Burst firing enhances neural output correlation. Front. Comput. Neurosci. 10:42. doi: 10.3389/fncom. 2016.00042

Cheong, E., Lee, S., Choi, B. J., Sun, M., Lee, C. J., and Shin, H. S. (2008). Tuning thalamic firing modes via simultaneous modulation of T- and L-type $\mathrm{Ca} 2+$ channels controls pain sensory gating in the thalamus. J. Neurosci. 28, 13331-13340. doi: 10.1523/JNEUROSCI.3013-08.2008

Cho, C.-H., Lee, S., Kim, A., Yarishkin, O., Ryoo, K., Lee, Y.-S., et al. (2020). TMEM16A expression in cholinergic neurons of the medial habenula mediates anxiety-related behaviors. EMBO Rep. 21:e48097. doi: 10.15252/embr. 201948097

Crocq, M.-A. (2015). A history of anxiety: from hippocrates to DSM. Dialogues Clin. Neurosci. 17, 319-325. doi: 10.31887/DCNS.2015.17.3/macrocq

Cueni, L., Canepari, M., Luján, R., Emmenegger, Y., Watanabe, M., Bond, C. T., et al. (2008). T-type Ca2+ channels, SK2 channels and SERCAs gate sleep-related oscillations in thalamic dendrites. Nat. Neurosci. 11, 683-692. doi: $10.1038 / \mathrm{nn} .2124$ 
Cui, Y., Yang, Y., Ni, Z., Dong, Y., Cai, G., Foncelle, A., et al. (2018). Astroglial Kir4.1 in the lateral habenula drives neuronal bursts in depression. Nature 554, 323-327. doi: 10.1038/nature25752

Deuschl, G., Raethjen, J., Lindemann, M., and Krack, P. (2001). The pathophysiology of tremor. Muscle Nerve 24, 716-735. doi: 10.1002/mus.1063

Dezsi, G., Ozturk, E., Stanic, D., Powell, K. L., Blumenfeld, H., O-Brien, T. J., et al. (2013). Ethosuximide reduces epileptogenesis and behavioral comorbidity in the GAERS model of genetic generalized epilepsy. Epilepsia 54, 635-643. doi: 10.1111/epi.12118

Dumenieu, M., Senkov, O., Mironov, A., Bourinet, E., Kreutz, M. R., Dityatev, A., et al. (2018). The low-threshold calcium channel Cav3.2 mediates burst firing of mature dentate granule cells. Cereb. cortex 28, 2594-2609. doi: 10.1093/cercor/bhy084

Dutton, A., and Dyball, R. E. (1979). Phasic firing enhances vasopressin release from the rat neurohypophysis. J. Physiol. 290, 433-440. doi: 10.1113/jphysiol. 1979.sp012781

Fisher, R. S., Acevedo, C., Arzimanoglou, A., Bogacz, A., Cross, J. H., Elger, C. E., et al. (2014). ILAE official report: a practical clinical definition of epilepsy. Epilepsia 55, 475-482. doi: 10.1111/epi.12550

Floresco, S. B., West, A. R., Ash, B., Moore, H., and Grace, A. A. (2003). Afferent modulation of dopamine neuron firing differentially regulates tonic and phasic dopamine transmission. Nat. Neurosci. 6, 968-973. doi: 10.1038/ nn1103

Fremont, R., Calderon, D. P., Maleki, S., and Khodakhah, K. (2014). Abnormal high-frequency burst firing of cerebellar neurons in rapid-onset dystoniaparkinsonism. J. Neurosci. 34, 11723-11732. doi: 10.1523/JNEUROSCI.140914.2014

Frotscher, M., and Léránth, C. (1985). Cholinergic innervation of the rat hippocampus as revealed by choline acetyltransferase immunocytochemistry: a combined light and electron microscopic study. J. Comp. Neurol. 239, 237-246. doi: 10.1002/cne.902390210

Gartside, S. E., Hajós-Korcsok, É., Bagdy, E., Hársing, L. G., Sharp, T., and Hajós, M. (2000). Neurochemical and electrophysiological studies on the functional significance of burst firing in serotonergic neurons. Neuroscience 98, 295-300. doi: 10.1016/s0306-4522(00)00060-9

Gerstner, W., Kistler, W. M., Naud, R., and Paninski, L. (2014). Neuronal Dynamics: From Single Neurons to Networks and Models of Cognition. Cambridge: Cambridge University Press, 442-446.

Gonon, F. G. (1988). Nonlinear relationship between impulse flow and dopamine released by rat midbrain dopaminergic neurons as studied by in vivo electrochemistry. Neuroscience 24, 19-28. doi: 10.1016/0306-4522(88)90307-7

Grace, A. A., and Bunney, B. S. (1984). The control of firing pattern in nigral dopamine neurons: burst firing. J. Neurosci. 4, 2877-2890. doi: 10.1523/JNEUROSCI.04-11-02877.1984

Haj-Dahmane, S., and Andrade, R. (1996). Muscarinic activation of a voltagedependent cation nonselective current in rat association cortex. J. Neurosci. 16, 3848-3861. doi: 10.1523/JNEUROSCI.16-12-03848.1996

Halassa, M. M., Siegle, J. H., Ritt, J. T., Ting, J. T., Feng, G., and Moore, C. I. (2011). Selective optical drive of thalamic reticular nucleus generates thalamic bursts and cortical spindles. Nat. Neurosci. 14, 1118-1120. doi: 10.1038/nn.2880

Handforth, A., Homanics, G. E., Covey, D. F., Krishnan, K., Lee, J. Y., Sakimura, K., et al. (2010). T-type calcium channel antagonists suppress tremor in two mouse models of essential tremor. Neuropharmacology 59, 380-387. doi: 10.1016/j. neuropharm.2010.05.012

Hildebrand, M. E., Isope, P., Miyazaki, T., Nakaya, T., Garcia, E., Feltz, A., et al. (2009). Functional coupling between mGluR1 and Cav3.1 T-type calcium channels contributes to parallel fiber-induced fast calcium signaling within Purkinje cell dendritic spines. J. Neurosci. 29, 9668-9682. doi: 10.1523/JNEUROSCI.0362-09.2009

Hornung, J. P. (2003). The human raphe nuclei and the serotonergic system. J. Chem. Neuroanat. 26, 331-343. doi: 10.1016/j.jchemneu.2003.10.002

Huerta, P. T., and Lisman, J. E. (1995). Bidirectional synaptic plasticity induced by a single burst during cholinergic theta oscillation in CA1 in vitro. Neuron 15 , 1053-1063. doi: 10.1016/0896-6273(95)90094-2

Iigaya, K., Minoura, Y., Onimaru, H., Kotani, S., and Izumizaki, M. (2019). Effects of feeding-related peptides on neuronal oscillation in the ventromedial hypothalamus. J. Clin. Med. 8:292. doi: 10.3390/jcm 8030292
Iigaya, K., Okazaki, S., Minoura, Y., and Onimaru, H. (2017). Interaction between novel oscillation within the ventromedial hypothalamus and the sympathetic nervous system. Neuroscience 343, 213-221. doi: 10.1016/j.neuroscience.2016. 11.048

Jagodic, M. M., Pathirathna, S., Nelson, M. T., Mancuso, S., Joksovic, P. M., Rosenberg, E. R., et al. (2007). Cell-Specific alterations of T-type calcium current in painful diabetic neuropathy enhance excitability of sensory neurons. J. Neurosci. 27, 3305-3316. doi: 10.1523/JNEUROSCI.4866-06.2007

Jiang, H., Fang, D., Kong, L.-Y., Jin, Z.-R., Cai, J., Kang, X.-J., et al. (2014). Sensitization of neurons in the central nucleus of the amygdala via the decreased GABAergic inhibition contributes to the development of neuropathic pain-related anxiety-like behaviors in rats. Mol. brain 7:72. doi: 10.1186/s13041-014-0072-Z

Joksimovic, S. M., Eggan, P., Izumi, Y., Joksimovic, S. L., Tesic, V., Dietz, R. M., et al. (2017). The role of T-type calcium channels in the subiculum: to burst or not to burst? J. Physiol. 595, 6327-6348. doi: 10.1113/JP274565

Jones, C., Barrera, I., Brothers, S., Ring, R., and Wahlestedt, C. (2017). Oxytocin and social functioning. Dialogues Clin. Neurosci. 19, 193-201. doi: $10.31887 /$ DCNS.2017.19.2/cjones

Jurek, B., and Neumann, I. D. (2018). The oxytocin receptor: from intracellular signaling to behavior. Physiol. Rev. 98, 1805-1908. doi: 10.1152/physrev.00031. 2017

Kang, H. W., Park, J. Y., Jeong, S. W., Kim, J. A., Moon, H. J., Perez-Reyes, E., et al. (2006). A molecular determinant of nickel inhibition in Cav3.2 T-type calcium channels. J. Biol. Chem. 281, 4823-4830. doi: 10.1074/jbc.M510 197200

Kawasaki, H., and Avoli, M. (1996). Excitatory effects induced by carbachol on bursting neurons of the rat subiculum. Neurosci. Lett. 219, 1-4. doi: 10.1016/S0304-3940(96)13175-X

Khosravani, H., Altier, C., Simms, B., Hamming, K. S., Snutch, T. P., Mezeyova, J., et al. (2004). Gating effects of mutations in the Cav3.2 T-type calcium channel associated with childhood absence epilepsy. J. Biol. Chem. 279, 9681-9684. doi: 10.1074/jbc.C400006200

Kim, D., Song, I., Keum, S., Lee, T., Jeong, M.-J., Kim, S.-S., et al. (2001). Lack of the burst firing of thalamocortical relay neurons and resistance to absence seizures in mice lacking $\alpha 1 \mathrm{G}$ T-type Ca2+ channels. Neuron 31, 35-45. doi: 10.1016/s0896-6273(01)00343-9

Kole, M. H. P., Bräuer, A. U., and Stuart, G. J. (2007). Inherited cortical HCN1 channel loss amplifies dendritic calcium electrogenesis and burst firing in a rat absence epilepsy model. J. Physiol. 578, 507-525. doi: 10.1113/jphysiol. 2006.122028

Kozlov, A. S., McKenna, F., Lee, J. H., Cribbs, L. L., Perez-Reyes, E., Feltz, A., et al. (1999). Distinct kinetics of cloned T-type Ca2 + channels lead to differential $\mathrm{Ca} 2$ + entry and frequency-dependence during mock action potentials. Eur. J. Neurosci. 11, 4149-4158. doi: 10.1046/j.1460-9568.1999. 00841.x

Kunwar, P. S., Zelikowsky, M., Remedios, R., Cai, H., Yilmaz, M., Meister, M., et al. (2015). Ventromedial hypothalamic neurons control a defensive emotion state. eLife 4:e06633. doi: 10.7554/eLife.06633

Lee, S., Lee, C., Woo, C., Kang, S. J., and Shin, K. S. (2019a). Chronic social defeat stress-induced enhancement of T-type calcium channels increases burstfiring neurons in the ventral subiculum. Biochem. Biophys. Res. Commun. 508, 1182-1187. doi: 10.1016/j.bbrc.2018.12.073

Lee, S., Lee, C., Woo, C., Kang, S. J., and Shin, K. S. (2019b). Chronic social defeat stress increases burst firing of nucleus accumbens-projecting ventral subicular neurons in stress-susceptible mice. Biochem. Biophys. Res. Commun. 515, 468-473. doi: 10.1016/j.bbrc.2019.05.180

Lenglet, S., Louiset, E., Delarue, C., Vaudry, H., and Contesse, V. (2002). Activation of 5-HT7 receptor in rat glomerulosa cells is associated with an increase in adenylyl cyclase activity and calcium influx through T-type calcium channels. Endocrinology 143, 1748-1760. doi: 10.1210/endo. 143.5.8817

Li, K., Zhou, T., Liao, L., Yang, Z., Wong, C., Henn, F., et al. (2013). BCaMKII in lateral habenula mediates core symptoms of depression. Science 341, 1016-1020. doi: 10.1126/science. 1240729

Lisman, J. E. (1997). Bursts as a unit of neural information: making unreliable synapses reliable. Trends Neurosci. 20, 38-43. doi: 10.1016/S01662236(96)10070-9 
Liu, X., Porteous, R., d-Anglemont de Tassigny, X., Colledge, W. H., Millar, R., Petersen, S. L., et al. (2011). Frequency-dependent recruitment of fast amino acid and slow neuropeptide neurotransmitter release controls gonadotropin-releasing hormone neuron excitability. J. Neurosci. 31, 2421-2430. doi: 10.1523/JNEUROSCI.5759-10.2011

Magee, J. C., Christofi, G., Miyakawa, H., Christie, B., Lasser-Ross, N., and Johnston, D. (1995). Subthreshold synaptic activation of voltage-gated Ca2+ channels mediates a localized Ca2+ influx into the dendrites of hippocampal pyramidal neurons. J. Neurophysiol. 74, 1335-1342. doi: 10.1152/jn.1995.74.3. 1335

Marks, W. N., Cain, S. M., Snutch, T. P., and Howland, J. G. (2016a). The T-type calcium channel antagonist Z944 rescues impairments in crossmodal and visual recognition memory in genetic absence epilepsy rats from strasbourg. Neurobiol. Dis. 94, 106-115. doi: 10.1016/j.nbd.2016.06.001

Marks, W. N., Greba, Q., Cain, S. M., Snutch, T. P., and Howland, J. G. (2016b). The T-type calcium channel antagonist Z944 disrupts prepulse inhibition in both epileptic and non-epileptic rats. Neuroscience 332, 121-129. doi: 10.1016/j. neuroscience.2016.06.035

McRory, J. E., Santi, C. M., Hamming, K. S., Mezeyova, J., Sutton, K. G., Baillie, D. L., et al. (2001). Molecular and functional characterization of a family of rat brain T-type calcium channels. J. Biol. Chem. 276, 3999-4011. doi: 10.1074/jbc.M008215200

Miwa, H., Koh, J., Kajimoto, Y., and Kondo, T. (2011). Effects of T-type calcium channel blockers on a parkinsonian tremor model in rats. Pharmacol. Biochem. Behav. 97, 656-659. doi: 10.1016/j.pbb.2010.11.014

Nelson, M. T., Joksovic, P. M., Su, P., Kang, H. W., Van Deusen, A., Baumgart, J. P., et al. (2007). Molecular mechanisms of subtype-specific inhibition of neuronal T-type calcium channels by ascorbate. J. Neurosci. 27, 12577-12583. doi: 10.1523/JNEUROSCI.2206-07.2007

Niday, Z., and Bean, B. P. (2021). BK channel regulation of afterpotentials and burst firing in cerebellar purkinje neurons. J. Neurosci. 41, 2854-2869. doi: 10.1523/JNEUROSCI.0192-20.2021

Padilla, S. L., Qiu, J., Soden, M. E., Sanz, E., Nestor, C. C., Barker, F. D., et al. (2016). Agouti-related peptide neural circuits mediate adaptive behaviors in the starved state. Nat. Neurosci. 19, 734-741. doi: 10.1038/nn.4274

Penn, D. L., Sanna, L. J., and Roberts, D. L. (2008). Social cognition in schizophrenia: an overview. Schizophr. Bull. 34, 408-411. doi: $10.1093 /$ schbul/sbn014

Perez-Reyes, E. (2003). Molecular physiology of low-voltage-activated T-type calcium channels. Physiol. Rev. 83, 117-161. doi: 10.1152/physrev.00018.2002

Petersen, A. V., Jensen, C. S., Crépel, V., Falkerslev, M., and Perrier, J. F. (2017). Serotonin regulates the firing of principal cells of the subiculum by inhibiting a T-type $\mathrm{Ca}(2+)$ current. Front. Cell. Neurosci. 11:60. doi: 10.3389/fncel.2017. 00060

Placantonakis, D. G., Schwarz, C., and Welsh, J. P. (2000). Serotonin suppresses subthreshold and suprathreshold oscillatory activity of rat inferior olivary neurones in vitro. J. Physiol. 524, 833-851. doi: 10.1111/j.1469-7793.2000. 00833.x

Prisco, S., Natoli, S., Bernardi, G., and Mercuri, N. B. (2002). Group I metabotropic glutamate receptors activate burst firing in rat midbrain dopaminergic neurons. Neuropharmacology 42, 289-296. doi: 10.1016/s0028-3908(01) 00192-7

Qiu, J., Bosch, M. A., Jamali, K., Xue, C., Kelly, M. J., and Ronnekleiv, O. K. (2006). Estrogen upregulates T-type calcium channels in the hypothalamus and pituitary. J. Neurosci. 26, 11072-11082. doi: 10.1523/JNEUROSCI.3229-06. 2006

Rangel, A., Sánchez-Armass, S., and Meza, U. (2010). Protein kinase C-mediated inhibition of recombinant T-type $\mathrm{Ca}_{v} 3.2$ channels by neurokinin 1 receptors. Mol. Pharmacol. 77, 202-210. doi: 10.1124/mol.109.058727

Reiner, A., and Levitz, J. (2018). Glutamatergic signaling in the central nervous system: ionotropic and metabotropic receptors in concert. Neuron 98, 1080-1098. doi: 10.1016/j.neuron.2018.05.018

Rouchet, N., Waroux, O., Lamy, C., Massotte, L., Scuvée-Moreau, J., Liégeois, J.F., et al. (2008). SK channel blockade promotes burst firing in dorsal raphe serotonergic neurons. Eur. J. Neurosci. 28, 1108-1115. doi: 10.1111/j.14609568.2008.06430.x

Rowley, N. M., Madsen, K. K., Schousboe, A., and Steve White, H. (2012). Glutamate and GABA synthesis, release, transport and metabolism as targets for seizure control. Neurochem. Int. 61, 546-558. doi: 10.1016/j.neuint.2012. 02.013

Sanabria, E. R., Su, H., and Yaari, Y. (2001). Initiation of network bursts by Ca2+dependent intrinsic bursting in the rat pilocarpine model of temporal lobe epilepsy. J. Physiol. 532, 205-216. doi: 10.1111/j.1469-7793.2001.0205g.x

Schultz, W. (1998). Predictive reward signal of dopamine neurons. J. Neurophysiol. 80, 1-27. doi: 10.1152/jn.1998.80.1.1

Schultz, W. (2002). Getting formal with dopamine and reward. Neuron 36, 241-263. doi: 10.1016/s0896-6273(02)00967-4

Sieber, A. R., Min, R., and Nevian, T. (2013). Non-Hebbian long-term potentiation of inhibitory synapses in the thalamus. J. Neurosci. 33, 15675-15685. doi: 10.1523/JNEUROSCI.0247-13.2013

Simonnet, J., and Brecht, M. (2019). Burst firing and spatial coding in subicular principal cells. J. Neurosci. 39, 3651-3662. doi: 10.1523/JNEUROSCI.1656-18. 2019

Splawski, I., Yoo, D. S., Stotz, S. C., Cherry, A., Clapham, D. E., and Keating, M. T. (2006). CACNA1H mutations in autism spectrum disorders. J. Biol. Chem. 281, 22085-22091. doi: 10.1074/jbc.M603316200

Tai, C.-H., Yang, Y.-C., Pan, M.-K., Huang, C.-S., and Kuo, C.-C. (2011). Modulation of subthalamic T-type $\mathrm{Ca}(2+)$ channels remedies locomotor deficits in a rat model of Parkinson disease. J. Clin. Invest. 121, 3289-3305. doi: $10.1172 /$ JCI46482

Tao, J., Hildebrand, M. E., Liao, P., Liang, M. C., Tan, G., Li, S., et al. (2008). Activation of corticotropin-releasing factor receptor 1 selectively inhibits CaV3.2 T-type calcium channels. Mol. Pharmacol. 73, 1596-1609. doi: 10.1124/mol.107.043612

Tirko, N. N., Eyring, K. W., Carcea, I., Mitre, M., Chao, M. V., Froemke, R. C., et al. (2018). Oxytocin transforms firing mode of CA2 hippocampal neurons. Neuron 100, 593-608. doi: 10.1016/j.neuron.2018.09.008

Tringham, E., Powell, K. L., Cain, S. M., Kuplast, K., Mezeyova, J., Weerapura, M., et al. (2012). T-type calcium channel blockers that attenuate thalamic burst firing and suppress absence seizures. Sci. Transl. Med. 4:121ra19. doi: 10.1126/scitranslmed.3003120

Vandael, D. H. F., Ottaviani, M. M., Legros, C., Lefort, C., Guérineau, N. C., Allio, A., et al. (2015). Reduced availability of voltage-gated sodium channels by depolarization or blockade by tetrodotoxin boosts burst firing and catecholamine release in mouse chromaffin cells. J. Physiol. 593, 905-927. doi: 10.1113/jphysiol.2014.283374

Vickstrom, C. R., Liu, X., Zhang, Y., Mu, L., Kelly, T. J., Yan, X., et al. (2020). Ttype calcium channels contribute to burst firing in a subpopulation of medial habenula neurons. eNeuro 7:ENEURO.0201-20.2020. doi: 10.1523/ENEURO. 0201-20.2020

Wang, X. J. (1999). Fast burst firing and short-term synaptic plasticity: a model of neocortical chattering neurons. Neuroscience 89, 347-362. doi: 10.1016/s03064522(98)00315-7

Wei, F., Yan, L. M., Su, T., He, N., Lin, Z. J., Wang, J., et al. (2017). Ion channel genes and epilepsy: functional alteration, pathogenic potential and mechanism of epilepsy. Neurosci. Bull. 33, 455-477. doi: 10.1007/s12264-017 $-0134-1$

Wolfe, J. T., Wang, H., Howard, J., Garrison, J. C., and Barrett, P. Q. (2003). T-type calcium channel regulation by specific G-protein betagamma subunits. Nature 424, 209-213. doi: 10.1038/nature01772

Womelsdorf, T., Ardid, S., Everling, S., and Valiante, T. A. (2014). Burst firing synchronizes prefrontal and anterior cingulate cortex during attentional control. Curr. Biol. 24, 2613-2621. doi: 10.1016/j.cub.2014.09.046

Yang, Y., Cui, Y., Sang, K., Dong, Y., Ni, Z., Ma, S., et al. (2018). Ketamine blocks bursting in the lateral habenula to rapidly relieve depression. Nature 554 , 317-322. doi: 10.1038/nature25509

Yuan, Y., Wu, W., Chen, M., Cai, F., Fan, C., Shen, W., et al. (2019). Reward inhibits paraventricular CRH neurons to relieve stress. Curr. Biol. 29, 1243.e4-1251.e4. doi: 10.1016/j.cub.2019.02.048

Zamponi, G. W., Bourinet, E., and Snutch, T. P. (1996). Nickel block of a family of neuronal calcium channels: subtype- and subunit-dependent action at multiple sites. J. Membr. Biol. 151, 77-90. doi: 10.1007/s002329900059

Zhang, C., Bosch, M. A., Rick, E. A., Kelly, M. J., and Rønnekleiv, O. K. (2009). 17Beta-estradiol regulation of T-type calcium channels in gonadotropin-releasing hormone neurons. J. Neurosci. 29, 10552-10562. doi: 10.1523/JNEUROSCI.2962-09.2009 
Zhang, L., Liu, Y., and Chen, X. (2005). Carbachol induces burst firing of dopamine cells in the ventral tegmental area by promoting calcium entry through L-type channels in the rat. J. Physiol. 568, 469-481. doi: 10.1113/jphysiol.2005.094722

Zhong, X., Liu, J. R., Kyle, J. W., Hanck, D. A., and Agnew, W. S. (2006). A profile of alternative RNA splicing and transcript variation of CACNA1H, a human T-channel gene candidate for idiopathic generalized epilepsies. Hum. Mol. Genet. 15, 1497-1512. doi: 10.1093/hmg/ ddl068

Conflict of Interest: The authors declare that the research was conducted in the absence of any commercial or financial relationships that could be construed as a potential conflict of interest.
Publisher's Note: All claims expressed in this article are solely those of the authors and do not necessarily represent those of their affiliated organizations, or those of the publisher, the editors and the reviewers. Any product that may be evaluated in this article, or claim that may be made by its manufacturer, is not guaranteed or endorsed by the publisher.

Copyright (C) 2021 Shao, Liu, Gao, Tu and Yang. This is an open-access article distributed under the terms of the Creative Commons Attribution License (CC BY). The use, distribution or reproduction in other forums is permitted, provided the original author(s) and the copyright owner(s) are credited and that the original publication in this journal is cited, in accordance with accepted academic practice. No use, distribution or reproduction is permitted which does not comply with these terms. 